\title{
Hospital Practice
}

\section{The practice of rectal examination}

\author{
D.P.K. Ng, J.F. Mayberry' ${ }^{1}$, A.S. McIntyre and R.G. Long \\ Medical Research Centre, City Hospital, Nottingham NG5 IPB and ${ }^{1}$ Leicester General Hospital, \\ Leicester, UK
}

\begin{abstract}
Summary: Rectal (both digital and rigid sigmoidoscopic) examination is an important part of the clinical examination. The aim of this study was to find out the opinions of the patients to this routine examination at the time of referral by their general practitioner and during their first hospital interview. Assessment of the value for the rectal examination was also examined.

We questioned 103 patients attending their second out-patient interview through a simple questionnaire. We discovered that patients considered awareness and explanation important: patients preferred to be told of the possibility of rectal examination prior to hospital consultation. Explanation of the method and reason was expected from hospital doctors. Formal consent was expected; informed verbal consent should be sufficient. Where logistically possible, a chaperone should always be present. Rectal examination would have facilitated the diagnosis in $47 \%$ of the patients examined. Thus, rectal examination should be performed on all patients where symptoms are referable to the lower gastrointestinal tract and where a possible diagnosis may be made or facilitated.
\end{abstract}

\section{Introduction}

There has been a recent renewal of interest in the practice of the rectal examination particularly the consequences of its oversight. ${ }^{1,2}$

Cultural and sexual attitudes on the part of the patient may result in a failure to examine the anus and rectum. The information obtained by rectal examination is important, particularly when symptoms are referable to the lower gastrointestinal tract. Over half of rectal cancers are palpable., ${ }^{1,3,4}$ Proctoscopy and/or rigid sigmoidoscopy allows direct visualization and biopsy of the mucosa, which is especially helpful in patients with inflammatory bowel disease. Performing rectal examination is thus beneficial for those where an abnormality is found. To determine patient opinion we questioned patients' attitudes to digital and sigmoidoscopic rectal examination.

\section{Patients and methods}

We surveyed 103 consecutive patients (age 15-81 years, median 48; 58 females) attending one of two general medical/gastroenterological out-patient

Correspondence: R.G. Long, M.D., F.R.C.P.

Accepted: 2 April 1991 clinics in a teaching hospital, by means of a questionnaire. Only those who had had both digital rectal examination (PR) and rigid sigmoidoscopy on their first visit were questioned. Digital and rigid sigmoidoscopic examinations were performed by one of four male doctors of senior house officer grade and above.

\section{Results (see Figure 1)}

There was a $100 \%$ response. Thirty-one patients with gastrointestinal symptoms had had a PR performed by their general practitioner (GP) prior to their hospital referral. Of the 73 who did not, 16 had diagnoses that would have been facilitated by PR (melaena for epigastric peptic ulcer pain and iron deficiency anaemia) and 33 had lesions that would have been revealed by proctoscopy or limited rigid sigmoidoscopy (inflammatory bowel disease, particularly 28 with ulcerative colitis).

Many patients wanted to be aware of the possible need for rectal examination before attending hospital, either to be told by their GP $(n=81)$ or in a letter accompanying their initial hospital appointment $(n=67)$. The large majority of patients wanted an explanation of how $(n=84)$, and why $(n=87)$ the procedure needed to be performed. Fifty-two felt that the examination required formal consent. Over half $(n=58)$ 
Information about the examination should be given by the general practitioner

An explanation of the need for the examination is important

Formal consent should be obtained before the examination

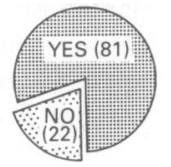

Information about the examination should be given in a letter from the hospital

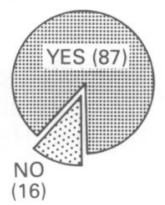

An explanation of the method is important

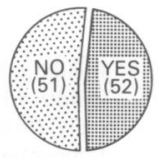

A chaperone should always be present during the examination
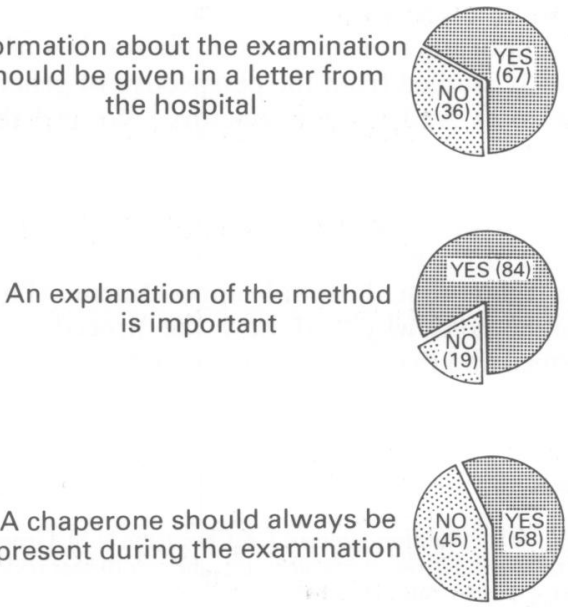

The numbers of GPs performing a rectal examination prior to hospital referral

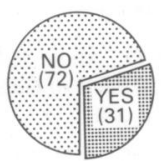

Figure 1 Results of the survey into patient opinion of the rectal examination.

thought that a chaperone was necessary. Twentytwo per cent of women and $25 \%$ of men would have preferred to be examined only by a doctor of the same sex. Seventy-one patients thought that rigid sigmoidoscopy was no more uncomfortable than PR. This did not depend on the seniority of the examiner.

\section{Discussion}

Any procedure which is performed without sedation naturally causes patient anxiety. In addition, rectal examination is relatively undignified. This can be diminished by the examiner developing a relaxed and composed attitude. This is enhanced by routinely explaining the procedure to patient in an attempt to alleviate anxiety and to improve patient co-operation.

The survey showed that patients wished to be informed before their hospital appointment of the possibility of having the examination performed. The large majority wanted this reiterated at their first hospital interview including an explanation of why the procedure was necessary.

Slightly greater than half the patients wanted formal consent. The area of consent is contentious, since it is implied when a patient seeks medical treatment at a clinic or surgery. The voluntary removal of clothing and lying on the examination couch in the left lateral position may be taken as consent. ${ }^{5}$ The main difficulty in undertaking this responsibility of implied consent is knowing how much information to give to the patient. ${ }^{6}$ This is discretionary, but it is generally acceptable that explanation of each manoeuvre of the procedure is given before it is performed.

Seventy-six patients did not mind being examined by a doctor of the opposite sex. However, 56 thought the examiner should always be accompanied by a chaperone. This represented the view of $58 \%$ of the female patients and $48 \%$ of the male, despite only male doctors participating during the period of the study.

Only 31 of our patients had been examined by their general practitioners. There are many factors involved to explain this, some of which were recently highlighted by Hennigan. ${ }^{2}$ This included the pressure of time, reluctance of the patient, the expectation that the examination was to be repeated, and not having a chaperone available. The latter is important both for patient reassurance, medico-legally ${ }^{7}$ and diagnostically (to help with instrumentation). Such an omission has been shown to lead to a delay in referral for resectable rectal malignancy. ${ }^{1}$ Nonetheless, $70 \%$ of low rectal carcinomas were detected by general practitioners and were appropriately referred. ${ }^{1}$ Our findings show that $P R$ with sigmoidoscopy is either diagnostic, or facilitates diagnosis, in $47 \%$. No patient in our study was found to have rectal cancer, which may be due to selection by GPs, 
resulting in direct surgical referral.

The importance of rectal examination has been previously studied from the perspective of general practitioners ${ }^{2}$ and hospital doctors. ${ }^{1}$ We feel this study is unique since we have surveyed the rectal examination from the patients' standpoint. We would therefore suggest that patients should be informed of the possibility of a rectal examination before their first hospital appointment. They should be told why and how the procedure is performed and that the examiner should be accom-

\section{References}

1. Dixon, A.R, Thornton-Holmes, J. \& Cheetham, N.M. General practitioners' awareness of colorectal cancer: a 10 year review. Br Med J 1990, 301: 152-153.

2. Hennigan, T.W., Franks, P.J., Hocken, D.B. \& Allen-Marsh, T.G. Rectal examination in general practice. $\mathrm{Br}$ Med $J 1990$, 301: $478-480$.

3. Williams, N.S. In: Bouchier, I.A.D., Allan, R.N., Hodgson, H.J.F., Keighley, M.R.B. (eds) Textbook of Gastroenterology. Baillière Tindall, London, 1984, p. 848. panied by a chaperone whenever possible. Verbal consent and cooperation of the patient to adopt an appropriate position probably provides sufficient consent. Rectal examination should be performed on all patients where a diagnosis might be made or facilitated by a rectal examination.

\section{Acknowledgement}

We gratefully acknowledge a grant from the Trent Regional Health Authority to $\mathrm{Dr} \mathrm{Ng}$

4. Fielding, L.P. \& Blesovsky, L. Clinical features of colorectal cancer. In: Misciewisc, J.J., Pounder, R.E. \& Venables, C.W. (eds) Diseases of the Gut and Pancreas. Blackwell Scientific Publications, Oxford, 1987, pp. 945-963.

5. Martin, C.R.A. Law Relating to Medical Practice, 2nd edition. Pitman Publishing, London, 1981.

6. Havard, J.D.J. Green Lectures. Br Med J 1989, 299: 503-504.

7. Medical Defence Union - Centenary Tales. Medical Defence Union, London, 1985. 